\title{
ARTICLE OPEN Brain gray matter network organization in psychotic disorders
}

\author{
Wenjing Zhang ${ }^{1}$, Du Lei ${ }^{1,2}$, Sarah K. Keedy ${ }^{3}$, Elena I. Ivleva ${ }^{4}$, Seenae Eum ${ }^{5}$, Li Yao ${ }^{1}$, Carol A. Tamminga ${ }^{4}$, Brett A. Clementz ${ }^{6}$, \\ Matcheri S. Keshavan ${ }^{7}$, Godfrey D. Pearlson ${ }^{8}$, Elliot S. Gershon $\mathbb{1}^{3}{ }^{3}$, Jeffrey R. Bishop ${ }^{5}$, Qiyong Gong (D) ${ }^{1}$, Su Lui ${ }^{1}$ and John A. Sweeney ${ }^{1,2}$
}

\begin{abstract}
Abnormal neuroanatomic brain networks have been reported in schizophrenia, but their characterization across patients with psychotic disorders, and their potential alterations in nonpsychotic relatives, remain to be clarified. Participants recruited by the Bipolar and Schizophrenia Network for Intermediate Phenotypes consortium included 326 probands with psychotic disorders (107 with schizophrenia (SZ), 87 with schizoaffective disorder (SAD), 132 with psychotic bipolar disorder (BD)), 315 of their nonpsychotic first-degree relatives and 202 healthy controls. Single-subject gray matter graphs were extracted from structural MRI scans, and whole-brain neuroanatomic organization was compared across the participant groups. Compared with healthy controls, psychotic probands showed decreased nodal efficiency mainly in bilateral superior temporal regions. These regions had altered morphological relationships primarily with frontal lobe regions, and their network-level alterations were associated with positive symptoms of psychosis. Nonpsychotic relatives showed lower nodal centrality metrics in the prefrontal cortex and subcortical regions, and higher nodal centrality metrics in the left cingulate cortex and left thalamus. Diagnosis-specific analysis indicated that individuals with SZ had lower nodal efficiency in bilateral superior temporal regions than controls, probands with SAD only exhibited lower nodal efficiency in the left superior and middle temporal gyrus, and individuals with psychotic BD did not show significant differences from healthy controls. Our findings provide novel evidence of clinically relevant disruptions in the anatomic association of the superior temporal lobe with other regions of whole-brain networks in patients with psychotic disorders, but not in their unaffected relatives, suggesting that it is a disease-related trait. Network disorganization primarily involving frontal lobe and subcortical regions in nonpsychotic relatives may be related to familial illness risk.
\end{abstract}

Neuropsychopharmacology (2020) 45:666-674; https://doi.org/10.1038/s41386-019-0586-2

\section{INTRODUCTION}

Efforts to identify imaging markers for psychotic disorders have been impeded by two factors: a focus on traditionally defined psychotic syndromes that have overlapping genetic, psychological, and neurobiological features [1-3], and a focus on regional changes such as gray matter volume $[4,5]$ or brain activity [6], or altered functional connectivity between pairs of regions [7, 8], rather than comprehensively on whole-brain networks that are believed to be fundamentally involved in the pathogenesis of psychosis [9-11]. Furthermore, prior studies in this area often utilized relatively small samples or post-hoc analyses of large data sets not collected with rigorous attention to consistency of image acquisition across ascertainment sites.

Phenotyping approaches grounded in network science can provide a more comprehensive understanding of neuropathological substrates of psychosis [10, 12,13]. Graph theory approaches based on functional connectivity and white matter connectivity have been used to characterize network graphs in patients with psychotic disorders [12, 14-17] and their relatives [18, 19]. However, the blood oxygen level-dependent (BOLD) signals reflecting brain function depend greatly on brain states [20, 21], and measurement of white matter pathways is affected by selection of tractography algorithms [22]. Approaches using brain gray matter anatomy to investigate brain networks in psychotic disorders may reveal more stable phenotypes related to altered anatomical organization [23-26]. The potential importance of this type of analysis for evaluating behaviorally relevant features of brain anatomy is reflected in observations that individual variation in morphometric similarity networks can account for $\sim 40 \%$ of the individual differences in IQ scores in healthy young people [25].

Previous studies characterized structural gray matter "connections" in schizophrenia patients $[27,28]$ and their offspring $[29,30]$ based on covariation in cortical gray matter measures such as volume or cortical thickness across the brain. Individuals with schizophrenia demonstrated a network abnormality with temporal rather than frontal hubs [27], and unaffected family members showed increased gray matter correlations within default-mode network regions [29]. However, in these analyses, gray matter networks were calculated by creating a whole-brain network for each group, thus individual networks for each participant could not be examined and related to clinical parameters of interest. An additional methodological issue is that studies mapping individual

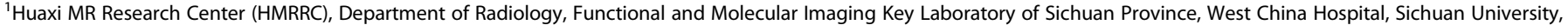

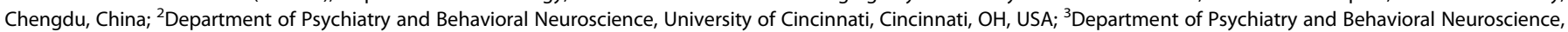

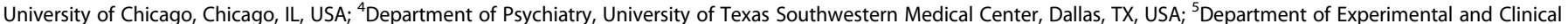

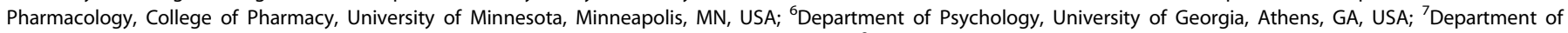

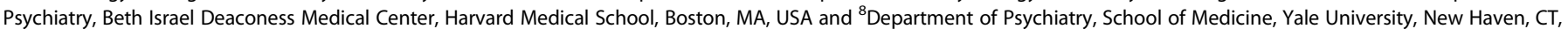
USA

Correspondence: Qiyong Gong (qygong05@126.com) or Su Lui (lusuwcums@tom.com) or John A. Sweeney (sweenej5@ucmail.uc.edu)
}

Received: 6 November 2019 Revised: 25 November 2019 Accepted: 30 November 2019

Published online: 7 December 2019 
brains into standard space before characterizing anatomic networks might have lost precision in quantitation of network topology from not taking the complex individualized folding structure of the brain into account. Notably, a recent study investigating the morphometric similarity across brain regions in patients with nonaffective psychoses demonstrated altered morphometric similarity in the frontal and temporal cortex, which were associated with brain expression of schizophrenia-related genes [23]. These previous findings support the potential of investigating anatomical brain networks across psychotic disorders and in nonpsychotic family members.

In this study, we generated single-subject gray matter graphs in psychotic individuals and their first-degree relatives with no history of psychosis collected by the Bipolar and Schizophrenia Network for Intermediate Phenotypes (B-SNIP) consortium. The primary analysis considered psychotic disorder patients combined across DSM diagnoses, based on growing evidence of overlapping cognitive, imaging, and genetic alterations across these conditions [31-34]. Secondary analyses were conducted for patients with each DSM diagnosis and their relatives.

\section{MATERIALS AND METHODS}

Participants

The B-SNIP consortium recruited participants across five sites in the United States that carried out parallel recruitment and phenotyping procedures as described previously [35]. We included 843 participants: 326 psychosis probands (107 with schizophrenia (SZ), 87 with schizoaffective disorder (SAD), and 132 with psychotic bipolar I disorder (BD)), 315 of their nonpsychotic first-degree relatives ( 120 of individuals with SZ, 83 of individuals with SAD, and 112 of individuals with psychotic BD), and 202 healthy participants (Table 1; Supplementary Table S1). Of patient probands included, all with artifact-free scans, $47.2 \%$ had at least one family member who had a MR scan performed and whose data met quality control (QC) standards discussed below. These relatives and their patient proband relative were included in within-family analyses described below. The study was approved by the Institutional Review Boards at each site, and all participants provided written informed consent prior to participation.

Probands were clinically stable and receiving consistent pharmacological treatment over the preceding month. The relatives included had no history of psychotic disorders. Axis I diagnoses in all groups were made based on the Structured Clinical Interview for DSM-IV Axis I Disorders and consensus case review. Diagnoses of Axis II disorders in relatives were made using the Structured Interview for DSM-IV Personality Disorders (SIDPIV). Exclusion criteria for all participants included: (1) contraindication to MRI scans, (2) substance abuse within past 1 month or substance dependence within 3 months, (3) significant systemic or neurological disorder, and (4) history of significant head trauma. Details regarding recruitment and clinical assessment strategies used by the B-SNIP consortium are available [33, 35].

MRI acquisition

High-resolution T1-weighted images were acquired using 3.0 T MRI scanners following guidelines of the Alzheimer's Disease Neuroimaging Initiative (ADNI) protocol (http://www.loni.ucla.edu/ ADNI/Research/Cores/) to enhance image consistency across sites/ scanners (Supplementary Table S2). Quantitative image quality ratings (IQR) were calculated for each subject and did not differ across participant groups (details in Supplementary Materials).

Extraction of brain networks

We followed the methodology proposed by Tijms et al. [36] to extract individual structural morphology brain networks, which is

Table 1. Demographic and clinical parameters for psychosis probands, their nonpsychotic relatives, and healthy controls.

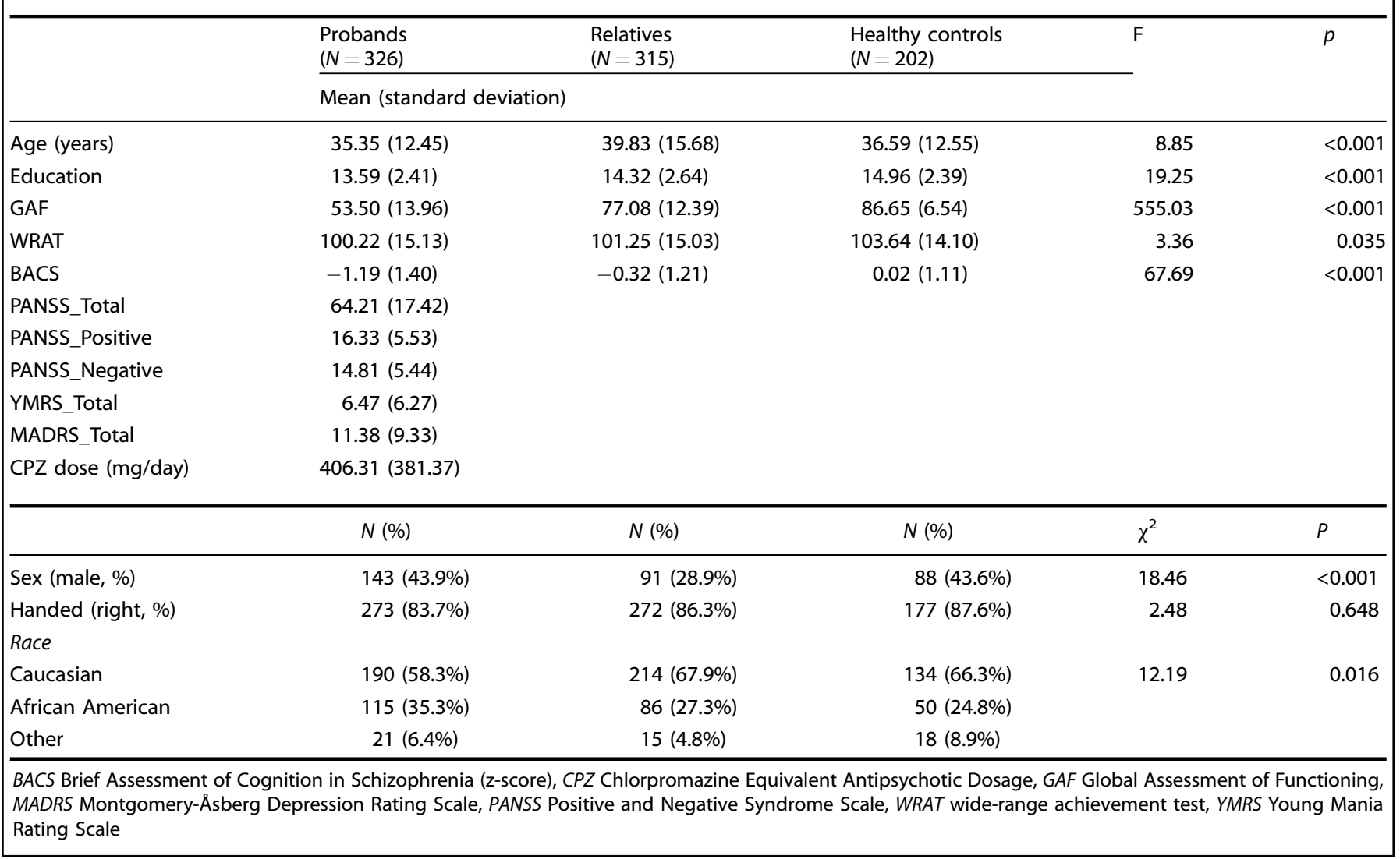


a completely automated and data-driven method. This approach takes both local morphology (e.g., thickness) and folding structure of the cortex into account, improving accuracy in the characterization of morphological network topology [24, 37]. In brief, the method defines network nodes as regions of interest (ROI) corresponding to $3 \times 3 \times 3 \mathrm{~mm}^{3}$ voxel cubes, and their "connection" refers to "edges", indicating statistically similar gray matter morphology of two cubes as determined with correlation coefficients. Weighted graphs are constructed after determining a threshold for each individual graph with a permutation-based method to ensure a significant similarity $(P<0.05)$ for all individuals [38]. Because network properties can vary with network size [39], we normalized gray matter networks using the methodology proposed by Batalle et al. [40] based on the Automated Anatomical Labeling (AAL) parcellation template [41] (details in Supplementary Materials).

Network properties

GRETNA (www.nitrc.org/projects/gretna/) was used to calculate topological properties of brain networks. A wide range of sparsity (S) thresholds was applied to all correlation matrices. S was determined to ensure that thresholded networks were estimable for the small-worldness scalar $(\sigma)$, defined as $\sigma>1.0$. Our threshold range was $0.05-0.40$ with an interval of 0.01 . This was determined using two criteria: (1) the average node of each threshold network on all nodes degree (node degree refers to the number of all edges connected to a node) is $>2 \times \log (N)$ (where $N$ is 90 , indicating the number of nodes); and (2) small-world scalar $\sigma$ of the threshold network of all subjects (as defined below) is $>1.1$ [42].

Global and nodal network properties were calculated at each sparsity threshold. Then, the area under the curve (AUC) was calculated for each network metric, providing a summarizing scalar for characterizing topological properties of brain networks independent of any single threshold selection as in previous studies [43, 44]. The following global metrics of small-world parameters were examined [42]: clustering coefficient $\left(C_{p}\right)$, characteristic path length $\left(L_{p}\right)$, normalized clustering coefficient $(\gamma)$, normalized characteristic path length $(\lambda)$, and small worldness $(\sigma)$. Network efficiency parameters, including local efficiency $\left(E_{\mathrm{loc}}\right)$ and global efficiency $\left(E_{\text {glob }}\right)[45]$, nodal centrality metrics including degree, efficiency, and betweenness, were also examined. Detailed explanation of each parameter is provided in Supplementary Materials.

Because the default-mode network (DMN), central executive network (CEN) and salience network (SN) have been shown to be altered in psychosis probands and their relatives [46-49], their gray matter topological network metrics were also calculated and compared among participant groups (Supplementary Materials).

Statistical analysis

Group comparison of network metrics. Analyses of covariance (ANCOVA) were performed to test for group differences in each network metrics (small-world, network efficiency, and nodal centrality measures) separately in psychosis probands and their nonpsychotic relatives in comparison with healthy controls. Site, age, sex, race, and handedness were included as covariates, and analyses used a false discovery rate (FDR) correction to preserve a $P<0.05$ experiment-wise threshold.

Abnormal network metrics found to be abnormal either in patient probands or relatives were examined separately in relation to DSM diagnoses of patient probands and experimental psychosis Biotypes defined by the B-SNIP consortium using a data-driven approach and cognitive, eye movement, and electroencephalographic (EEG) measures [3]. Findings from these analyses are shown in Supplementary Materials.

Within-family correlations. After excluding patient and relative cases based on QC of the MRI data or relatives being unwilling or unable to have MRI scans, $47 \%$ of the patient probands had a family member with valid MRI data. To analyze patients and relatives together considering their family relationship, we included these patients and relatives and the controls with ANCOVAs as above to identify group differences, treating family membership as a random effect. Second, we examined the correlation of the data from these patients and their specific relatives, and within diagnoses for all global network measures and all metrics in which differences were seen in comparisons of relatives or probands vs. healthy controls. Site, age, sex, race, and handedness were treated as covariates in these analyses. Findings are shown in Supplementary Materials.

Network matrix comparisons of abnormal nodes. The correlation matrices of inter-region associations of regions with altered nodal characteristics were examined to identify regions with altered connection to aberrant nodes found in psychotic probands or their nonpsychotic relatives in comparison with correlations in controls using the network-based statistics (NBS) method [50]. Findings are shown in Supplementary Materials.

Correlations with clinical variables. Structural network metrics that differed between probands and controls were correlated with psychiatric symptom severity ratings using the Positive and Negative Syndrome Scale (PANSS) [51], Young Mania Rating Scale (YMRS) [52], Montgomery-Åsberg Depression Rating Scale (MADRS) [53], and the Global Assessment of Functioning (GAF) scale, adjusting for site, age, sex, race, and handedness. We also examined associations with general cognitive functioning as assessed by the Brief Assessment of Cognition in Schizophrenia (BACS) scale [31]. In nonpsychotic relatives, altered network metrics were correlated with the presence of psychosis spectrum (Cluster A) personality features (within 1 criterion of diagnosis). Since the relative sample includes younger individuals still in the risk period, age effects on altered network metrics were also examined. Nominal significance thresholds were used for these exploratory/heuristic analyses.

\section{RESULTS}

Topological metrics of brain gray matter networks

All patient probands, their nonpsychotic relatives, and healthy controls showed a small-world architecture (i.e., $\sigma>1$ ) at all connection densities. There were no significant differences in psychosis probands or their nonpsychotic relatives compared with healthy controls in global network properties, including global/ local efficiency, clustering coefficient, characteristic path length, normalized clustering coefficient, or normalized characteristic path length.

Relative to healthy controls, probands with psychotic disorders showed reduced nodal efficiency in the left superior temporal gyrus (STG), left middle temporal gyrus (MTG), and bilateral superior temporal pole (STP), implying reduced capacity for propagating information with other brain nodes. Compared with healthy controls, nonpsychotic relatives had significantly lower nodal degree (fewer nodal connections) in the right middle frontal gyrus (MFG), right orbital inferior frontal gyrus (IFG), left hippocampus, left pallidum, and higher nodal degree in the left posterior cingulate gyrus (PCG) and left thalamus. Nonpsychotic relatives also showed significantly lower nodal efficiency in right MFG, right orbital IFG, left hippocampus, and higher nodal efficiency in the left PCG and left thalamus compared with healthy controls (all with FDR corrected $P<0.05$, Fig. 1, Table 2). All of these findings remained significant after IQR was treated as an additional covariate.

In the subgroup of patients and relatives from the same families, ANCOVAs were performed as above, but treating family membership as a random effect. These analyses identified significant group differences among probands, nonpsychotic relatives, and healthy controls in nodal efficiency in the left STG, 

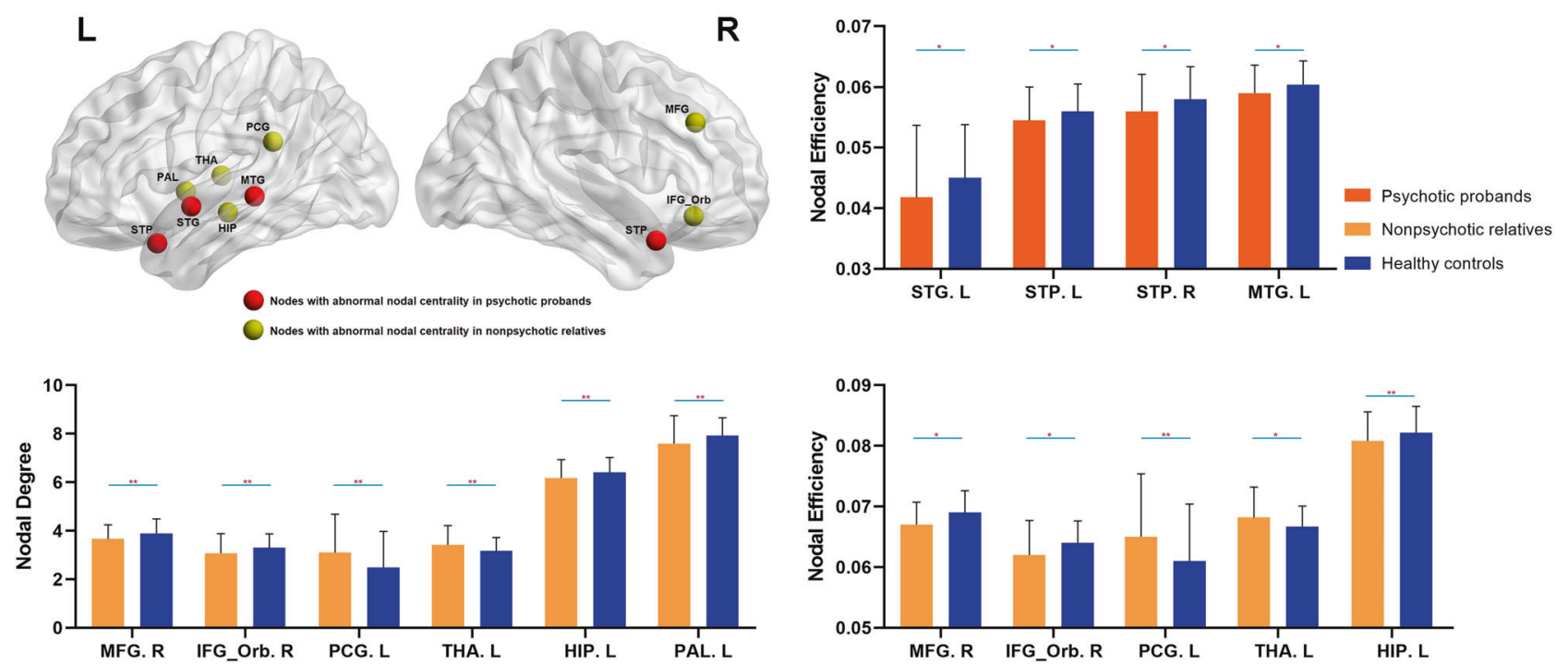

Fig. 1 Intergroup comparisons of gray matter network metrics between psychotic probands or their nonpsychotic relatives and healthy controls. ${ }^{* *} P<0.01 ; * P<0.05$. HIP hippocampus, MFG middle frontal gyrus, MTG middle temporal gyrus, IFG_Orb orbital inferior frontal gyrus, PCG posterior cingulate gyrus, PAL pallidum, STG superior temporal gyrus, STP superior temporal pole, THA thalamus, L left, R right.

left MTG, and bilateral STP, as well as left PCG and left thalamus. Differences in nodal degree were observed in the right orbital IFG, left PCG, and left thalamus. Direct comparison of psychosis probands with their nonpsychotic relatives from same families can be informative regarding familial and disease associations, as their background genetic and environmental factors are more similar than in the general population. Probands showed lower nodal degree/efficiency compared with their relatives in regions where alterations were seen in comparison of probands with healthy controls in the primary analyses (lower nodal efficiency of the left STG, left MTG, and bilateral STP). Also, in this analysis with better control of background familial factors, we identified an additional alteration of higher nodal degree and efficiency in the left thalamus in relatives compared with probands after FDR correction, similar to the differences between relatives and controls.

\section{Within-family correlations}

In regions with altered anatomic similarity metrics of patient probands or relatives, four out of sixteen had significant familial association. Within the four temporal lobe alterations observed in probands, only the left STG showed modestly significant familiality. In the 11 alterations observed in relatives, nodal degree of the left thalamus and nodal efficiency of the left hippocampus and left thalamus showed significant familiality (Table 3). Disorder-specific analyses indicated that two of these measures were significant in SAD patients, one of these in SZ patients as well, and none in bipolar patients. None of the four temporal lobe metrics altered in patient probands showed significant familiality in any DSM disorder. Detailed findings are shown in Supplementary Materials.

Network matrix of abnormal nodes

Inspection of correlations of abnormal temporal lobe areas with other brain regions indicated atypical anatomic similarity profiles with prefrontal regions and several subcortical regions relative to the correlations of these brain regions in the healthy controls (Supplementary Table S4). Similar analyses were conducted in regions with altered anatomic similarity profiles for the relatives (Supplementary Table S5).

Comparisons of altered network metrics in patient diagnostic subgroups

Network metrics found to be abnormal in the whole proband group were compared separately for each proband DSM diagnostic group vs. controls. Individuals with SZ showed lower nodal efficiency in the left STG, left MTG, and bilateral STP. Probands with SAD exhibited lower nodal efficiency in the left STG and left MTG. Individuals with psychotic BD did not differ from healthy controls. These findings were significant after FDR correction (Fig. 2; Supplementary Table S6). While this pattern is consistent with continuum models of psychotic disorder severity from $S Z$ to $S A D$ to $B D$, we note that no significant differences were observed among patient groups with different DSM diagnoses across network metrics. Alter network metrics in patients sorted by previously reported B-SNIP experimental Biotypes defined by cognitive and neurophysiological parameters from the same overall patient sample are presented in Supplementary Materials (Supplementary Table S7). Notably, the group with the greatest cognitive and neurophysiological alteration (Biotype 1) had the most significant alterations in temporal lobe metrics. In direct comparison of patients with different Biotypes, no significant group differences were observed.

\section{Correlation with clinical ratings}

In exploratory analyses conducted with nominal significance thresholds, reductions in nodal efficiency of the left STG and bilateral STP were associated with higher PANSS-positive symptom scores in psychosis probands, and reductions in nodal efficiency of left MTG were associated with higher PANSS-positive symptom scores and lower GAF scores (Supplementary Fig. S1). No associations of temporal lobe nodal alterations were observed with PANSS total or negative symptom scores or the individual hallucination/delusion items, or with neuropsychological deficit (BACS scores) or daily antipsychotic dose in probands (in chlorpromazine equivalents) $(P>0.05)$.

In nonpsychotic relatives, no significant correlations were observed between any altered network metric with BACS data or the presence of psychosis spectrum personality features. The nodal degree $(r=-0.34, P<0.001)$ and nodal efficiency $(r=$ $-0.38, P<0.001)$ measures in the right MFG showed significant age-related decline, but these relations did not differ significantly from those of healthy controls (Supplementary Materials).

\section{DISCUSSION}

By investigating single-subject graphs reflecting the structure of gray matter network morphology in individuals with psychotic 
Table 2. Network metrics of regions showing significant intergroup differences in psychotic probands and their nonpsychotic relatives.

\begin{tabular}{|c|c|c|c|c|c|}
\hline \multirow[t]{2}{*}{ Regions with altered network metrics } & \multirow[t]{2}{*}{ Nodal centrality metrics } & \multirow{2}{*}{$\begin{array}{l}\text { Probands } \\
(N=326) / \text { relatives }(N=315) \\
\text { Mean } \pm \text { SD }\end{array}$} & \multirow{2}{*}{$\begin{array}{l}\mathrm{HC}(N=202) \\
\text { Mean } \pm \mathrm{SD}\end{array}$} & \multicolumn{2}{|c|}{ ANCOVA } \\
\hline & & & & $\mathrm{F}$ & $P^{*}$ \\
\hline \multicolumn{6}{|l|}{ Psychotic probands vs healthy controls } \\
\hline STG, L & Efficiency & $0.042 \pm 0.012$ & $0.045 \pm 0.009$ & 11.36 & 0.024 \\
\hline STP, L & Efficiency & $0.055 \pm 0.006$ & $0.056 \pm 0.004$ & 10.37 & 0.031 \\
\hline STP, R & Efficiency & $0.056 \pm 0.006$ & $0.058 \pm 0.005$ & 11.99 & 0.024 \\
\hline MTG, L & Efficiency & $0.059 \pm 0.005$ & $0.060 \pm 0.004$ & 9.55 & 0.038 \\
\hline \multicolumn{6}{|l|}{ Nonpsychotic relatives vs healthy controls } \\
\hline MFG, R & Degree & $3.67 \pm 0.58$ & $3.89 \pm 0.60$ & 10.43 & 0.009 \\
\hline IFG_Orb, R & Degree & $3.07 \pm 0.81$ & $3.30 \pm 0.57$ & 12.93 & 0.006 \\
\hline PCG, L & Degree & $3.11 \pm 1.58$ & $2.49 \pm 1.49$ & 17.17 & 0.004 \\
\hline Hippocampus, L & Degree & $6.18 \pm 0.75$ & $6.40 \pm 0.62$ & 12.57 & 0.006 \\
\hline Pallidum, L & Degree & $7.58 \pm 1.16$ & $7.93 \pm 0.73$ & 12.42 & 0.006 \\
\hline Thalamus, L & Degree & $3.42 \pm 0.79$ & $3.17 \pm 0.55$ & 12.39 & 0.006 \\
\hline MFG, R & Efficiency & $0.067 \pm 0.004$ & $0.069 \pm 0.004$ & 9.52 & 0.013 \\
\hline IFG_Orb, R & Efficiency & $0.062 \pm 0.006$ & $0.064 \pm 0.004$ & 9.07 & 0.018 \\
\hline PCG, L & Efficiency & $0.065 \pm 0.010$ & $0.061 \pm 0.009$ & 14.12 & 0.003 \\
\hline Hippocampus, L & Efficiency & $0.081 \pm 0.005$ & $0.082 \pm 0.004$ & 14.31 & 0.003 \\
\hline Thalamus, L & Efficiency & $0.068 \pm 0.005$ & $0.067 \pm 0.003$ & 9.12 & 0.018 \\
\hline
\end{tabular}

ANCOVA analysis of covariance, FDR false discovery rate, $H C$ healthy controls, $S D$ standard deviation, MFG middle frontal gyrus, MTG middle temporal gyrus, IFG_Orb orbital inferior frontal gyrus, PCG posterior cingulate gyrus, STG superior temporal gyrus, STP superior temporal pole, $L$ left, $R$ right " $P$-values that were corrected with FDR

Table 3. Within-family correlation of network metrics that had significant intergroup differences among the participant groups.

\begin{tabular}{|c|c|c|c|c|c|}
\hline \multirow{2}{*}{$\begin{array}{l}\text { Regions with altered network } \\
\text { metrics }\end{array}$} & \multirow{2}{*}{$\begin{array}{l}\text { Nodal centrality } \\
\text { metrics }\end{array}$} & \multirow{2}{*}{$\begin{array}{l}\text { Overall } \\
\text { probands }\end{array}$} & \multicolumn{3}{|c|}{ DSM disorders } \\
\hline & & & $\mathrm{SZ}$ & SAD & $\mathrm{BD}$ \\
\hline \multicolumn{6}{|c|}{ Network metrics altered in psychotic probands } \\
\hline STG, L & Efficiency & $\begin{array}{l}r=0.25 \\
P=0.009\end{array}$ & / & / & / \\
\hline STP, L & Efficiency & / & / & / & / \\
\hline STP, R & Efficiency & / & / & / & / \\
\hline MTG, L & Efficiency & / & / & / & / \\
\hline \multicolumn{6}{|c|}{ Network metrics altered in nonpsychotic relatives } \\
\hline MFG, R & Degree & / & / & / & / \\
\hline IFG_Orb, R & Degree & / & / & / & I \\
\hline PCG, L & Degree & / & / & / & / \\
\hline Hippocampus, L & Degree & / & / & / & / \\
\hline Pallidum, L & Degree & / & / & / & I \\
\hline Thalamus, L & Degree & $\begin{array}{l}r=0.39 \\
P<0.001\end{array}$ & / & $\begin{array}{l}r= \\
0.55 \\
P \\
=0.00\end{array}$ & / \\
\hline MFG, R & Efficiency & / & / & / & I \\
\hline IFG_Orb, R & Efficiency & / & / & / & / \\
\hline PCG, L & Efficiency & / & / & / & l \\
\hline Hippocampus, L & Efficiency & $\begin{array}{l}r=0.23 \\
P=0.018\end{array}$ & / & / & / \\
\hline Thalamus, L & Efficiency & $\begin{array}{l}r=0.41 \\
P<0.001\end{array}$ & $\begin{array}{l}r= \\
0.44 \\
P= \\
0.017\end{array}$ & $\begin{array}{l}r= \\
0.58 \\
P= \\
0.001\end{array}$ & / \\
\hline
\end{tabular}

$B D$ psychotic bipolar I disorder, $F D R$ false discovery rate, $S A D$ schizoaffective disorder, $S Z$ schizophrenia, MFG middle frontal gyrus, MTG middle temporal gyrus, IFG_Orb orbital inferior frontal gyrus, PCG posterior cingulate gyrus, STG superior temporal gyrus, STP superior temporal pole, $L$ left, $R$ right

${ }^{*} P$-values listed in the table were corrected using FDR disorders and their first-degree relatives, we found that psychotic probands demonstrated clinically relevant decreased nodal efficiency in bilateral temporal lobes, mainly in the superior temporal cortex and temporal poles, relative to healthy individuals. Comparison of patients with their own nonpsychotic relatives revealed similar findings and an additional network alteration in the left thalamus. Nonpsychotic relatives, compared with community controls, showed lower nodal degree in the right prefrontal cortex, left pallidum, and left hippocampus, and higher nodal degree in the left posterior cingulate cortex and left thalamus with mostly parallel alterations of nodal efficiency in these regions. These findings in a study conducted by a multisite consortium with rigorous attention to consistency of image acquisition and clinical assessment provides evidence for the disruptions of superior temporal cortex in case probands but not in unaffected relatives, with minimal familiality of these alterations, suggesting that they may represent disease-related traits associated with psychotic illness. Altered anatomic connection mainly involving the prefrontal cortex, hippocampus, and striatum in relatives may be related to illness risk.

Gray matter network alterations in probands

Network disorganization involving the superior and middle temporal cortex was observed in patients in comparison with their first-degree relatives and community controls. This alteration was associated with the level of residual psychosis symptom severity in our clinically stable psychosis proband cohort. Superior temporal regions include primary and secondary auditory cortices and areas related to language processing, while MTG is involved in semantic memory and language processes [54,55]. These regions have been reported to have reduced local functional connectivity or abnormal network hubs in psychotic individuals using graph analysis [17, 56, 57]. Regional abnormalities in the superior temporal cortex have also been reported with both functional [20] and structural analysis $[58,59]$ in patients with psychotic disorders, which have been related to positive symptoms [59], 

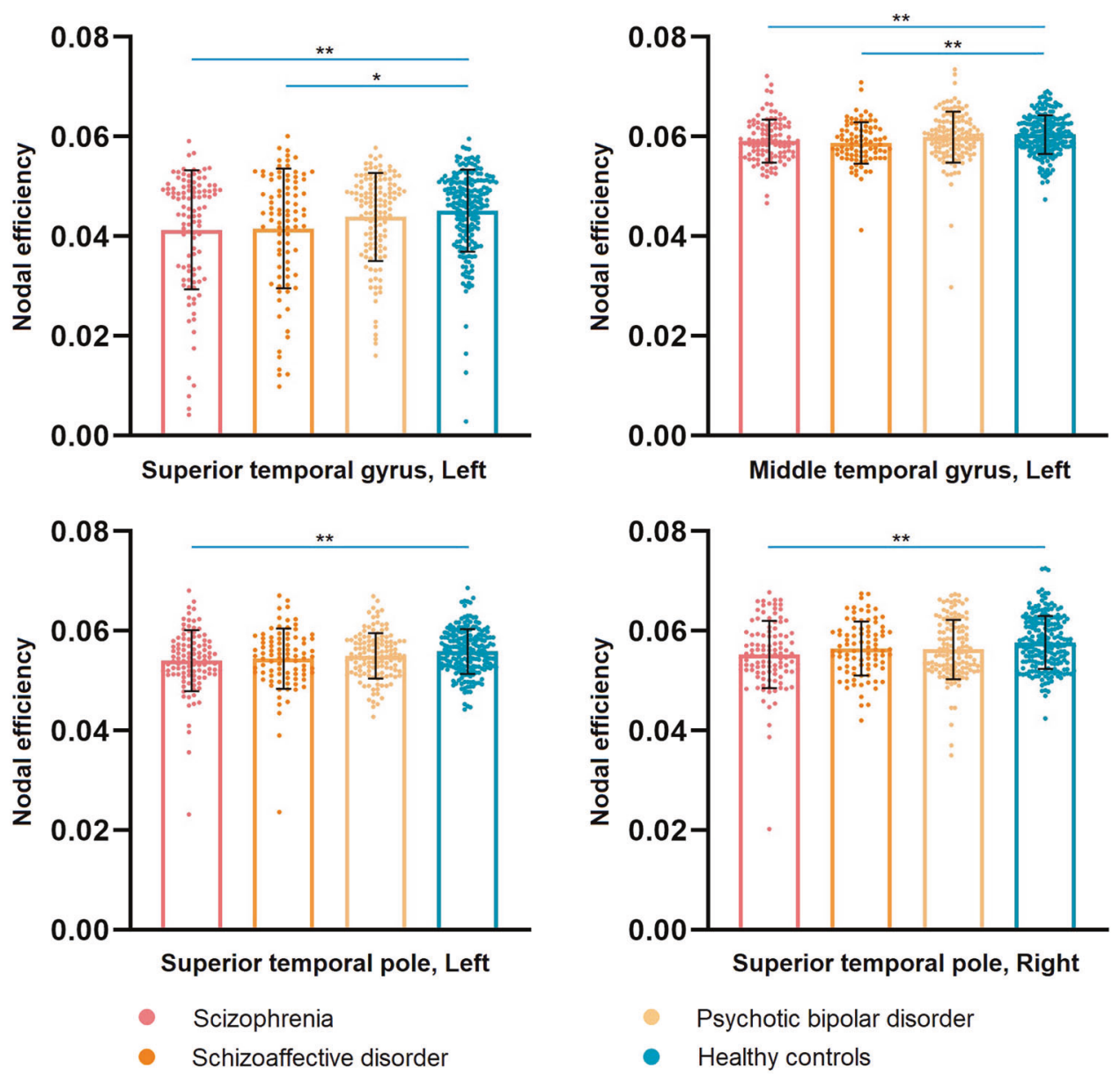

Fig. 2 The pair-wise comparisons of selected nodal metrics shown to be altered in the whole proband group between individuals with each DSM diagnosis and healthy controls. ${ }^{* *} P<0.01 ; * P<0.05$

including auditory hallucinations $[60,61]$. These findings suggest an important role of the superior temporal cortex in the neuropathology of psychotic disorders.

It is important to emphasize that our findings of abnormal brain network organization in the superior temporal cortex reflect an alteration in its morphometric characteristics in relation to those of other brain areas. Our supplementary node-to-node correlational studies revealed that the most notable and consistent factors influencing altered nodal centrality of superior temporal regions involved alterations in its anatomic relationship with features of the prefrontal cortex and subcortical regions, including amygdala and striatum. Regional structural abnormalities and functional disconnectivity within frontotemporal circuits have been reported in psychotic illnesses in several studies [4, 62-64], and an altered relationship between temporal lobe function and striatal dopamine storage/synthesis capacity has been related to psychosis vulnerability [65]. Together with previous evidence from structural similarity and covariation network analyses in nonaffective psychoses $[23,27,66]$, our findings support the notion of clinically relevant network hub abnormalities of superior temporal regions in psychotic disorders.

Gray matter network alterations in relatives

In nonpsychotic first-degree relatives, we observed lower nodal efficiency in the right prefrontal cortex and left hippocampus and pallidum compared with healthy controls, suggesting a reduced use or capacity of these regions for propagating information to other nodes. These results parallel previous findings of lower clustering coefficient values and regional gray matter deficits in right frontal regions $[66,67]$, as well as functional and structural abnormalities in the hippocampus $[68,69]$, in individuals with familial risk for psychotic disorders. The impaired functional integration between the posterior hippocampus and prefrontal cortex has also been found in individuals at high risk for psychosis [70].

Nonpsychotic relatives also showed higher nodal centrality metrics in the left posterior cingulate cortex and left thalamus relative to healthy controls. Higher nodal centrality metrics suggest an enhanced connection of the node with others in the brain network configuration. Recent work investigating graph topology in relatives of SZ patients also indicated a higher nodal efficiency in the posterior cingulate cortex [71]. In a previous functional study defining gray matter "connections" using volumetric covariation, increased gray matter correlations with the posterior cingulate cortex were found in family members of SZ patients [29]. While previous gray matter volumetric analysis with B-SNIP participants indicated no significant abnormalities in nonpsychotic relatives [4], our network-based analysis may be more sensitive to patterns of gray matter changes, supporting the idea that anatomic graph analysis represents a promising approach for characterizing subtler neuroanatomic alterations associated with risk for psychotic disorders in the context of whole-brain anatomic configuration.

Gray matter network differences between probands and relatives The pattern of altered anatomic association of the superior temporal cortex in brain networks of probands contrasts with primarily cortical involvement of the frontal lobe in relatives. This 
represents a significant novel finding. In an investigation using a similar analytic approach, robust gray matter network-based changes in temporal regions were also not seen in relatives of schizophrenia probands [66]. Prior studies performing regional measurements of superior temporal cortex anatomy in unaffected siblings of patients with psychotic disorder also have not found evidence of superior temporal alterations $[72,73]$. Thus, alterations in brain network organization involving superior temporal cortex appears to be associated with neuropathological manifestations of psychotic illness, while altered connection of the frontal cortex into brain networks in relatives may be related to familial illness risk.

This pattern of findings requires consideration of why a putative illness risk marker in unaffected relatives would be less robustly expressed in affected patients. While there is much evidence of frontal lobe alteration in schizophrenia, it is possible that illnessrelated changes in the temporal cortex and its connections with the frontal cortex as observed in our patients, or drug treatment effects on frontostriatal circuitry $[74,75]$, may variably affect frontal lobe connectivity in patients, reducing a more consistent pattern of frontal lobe connectivity alteration seen in nonpsychotic family members. While mechanisms for this difference between patients and unaffected relatives requires further study, our findings are consistent with the view that changes in STG are more related to being affected with a psychotic disorder than to familial factors, and thus may better serve as an illness than a risk biomarker.

\section{DSM diagnosis and B-SNIP biotype-specific analyses}

In exploratory analysis, while patients with different DSM diagnoses did not differ in network metrics, changes in patients with SZ replicated all the network alterations as seen in the combined sample, while BD patients did not demonstrate any significant alteration. These analyses support continuum models for brain alterations across psychotic disorders, in which SZ and $\mathrm{BD}$ stand at the distant ends of a severity continuum of imaging deficits [1, 3], consistent with previous structural [4, 33] and resting-state fMRI [76] studies of regional brain features. A similar pattern of dimensional effects across disorders has been seen in heritability estimates of susceptibility genes [77], neuropsychological deficits [31], and clinical ratings [35, 78]. Network changes of patients with different B-SNIP biotypes were also in line with previous observations using other MR metrics, indicating Biotype 1 patients present with the most severe brain alterations while Biotype 3 patients have minimal brain alterations [3].

\section{Limitations}

There are potential limitations that need to be considered when interpreting the current findings. First, this study employed anatomical templates to ensure the same number of nodes across individuals and enforce identical connectivity density to facilitate network comparisons, as in most previous studies. While this has limitations, we note that graphs for participants were analyzed in their native space, preserving inter-individual variability. Second, the potential confounding effects of antipsychotic medication on brain measures cannot be ruled out, though correlations of our findings with current antipsychotic drug dose were not significant. Third, although the spatial resolution of our data is comparable with that used in previous gray matter network analyses [36, 79, 80], higher resolution data acquisition in the future may increase precision of findings. Fourth, in the case of relatives, it is unclear whether the alterations reflect familial pathology or are linked to resilience and the absence of psychosis in this population. Prospective studies are needed to resolve this issue. Fifth, some previous studies adopted morphometric variables obtained from both T1-weighted imaging and diffusion-weighted imaging to map morphometric similarity networks [23, 25]. Future work with multimodal MRI may ultimately provide more precise morphometric network characterization as strategies for bringing these approaches into spatial alignment are better developed. Sixth, while our findings highlight the importance of STG and temporal pole alterations in anatomic network alterations in psychotic disorders, their relation to functional STG network integration remains to be determined. Finally, psychosis diagnoses were limited to common psychotic disorders, including SZ, SAD, and psychotic BD, not the full psychosis spectrum, and imaging studies were completed outside of acute episodes, potentially limiting clinical-pathological correlations.

\section{FUNDING AND DISCLOSURE}

This work was supported by the National Institute of Mental Health Grant Nos. MH077851 (to CAT), MH078113 (to MSK), MH077945 (to GDP), MH077852 (to Gunvant K. Thaker), and MH077862 (to JAS). The National Institute of Mental Health had no further role in study design; in the collection, analysis, and interpretation of the data; in the writing of the report; and in the decision to submit the paper for publication. The work was also supported by the National Natural Science Foundation of China (81671664, 81820108018, and 81621003), Miaozi Project in Science and Technology Innovation Program of Sichuan Province and 1.3.5 Project for Disciplines of Excellence, West China Hospital, Sichuan University (Project No. ZYYC08001, ZYJC18020). CAT has served on the advisory board for drug development for Intra-Cellular Therapies, Inc., as an ad hoc consultant for Eli Lilly, Sunovion, Astellas, Pfizer, and Merck, has been a council member and unpaid volunteer for the National Alliance on Mental Illness, and served as deputy editor for the American Psychiatric Association. MSK has received research support from Sunovion and GlaxoSmithKline. Remaining authors report no competing interests.

\section{ACKNOWLEDGEMENTS}

We thank Gunvant K. Thaker, MD, at the Maryland Psychiatric Research Institute, University of Maryland, who was closely involved with the initial stages of the Bipolar-Schizophrenia Network for Intermediate Phenotypes consortium development and its conceptual and methodological aspects. We acknowledge the many staff members from the B-SNIP consortium who assisted in the collection of brain images and performed clinical assessments, and thank participants for their generous contributions in time and effort to our project.

\section{AUTHOR CONTRIBUTIONS}

JRB, ESG, QG, SL and JAS contributed to the conception and design of the study. WZ, $D L, L Y$ and JAS contributed to the statistical analysis. All authors contributed to the acquisition, or analysis and interpretation of the data. WZ, DL, JRB, SL and JAS contributed to the drafting of the paper, while all authors made critical revision of the paper for important intellectual content and gave final approval of the version to be published. CAT, MSK, GDP, QG, SL and JAS obtained funding to support this work. WZ and JAS had full access to all the data of the study and take responsibility for the integrity of the data and the accuracy of the data analysis. JAS, SL and QG contributed equally to work on this project.

\section{ADDITIONAL INFORMATION}

Supplementary Information accompanies this paper at (https://doi.org/10.1038/ s41386-019-0586-2)

Publisher's note Springer Nature remains neutral with regard to jurisdictional claims in published maps and institutional affiliations. 


\section{REFERENCES}

1. Guloksuz S, van Os J. The slow death of the concept of schizophrenia and the painful birth of the psychosis spectrum. Psychol Med. 2018;48:229-44.

2. Schwarz E, Doan NT, Pergola G, Westlye LT, Kaufmann T, Wolfers T, et al. Reproducible grey matter patterns index a multivariate, global alteration of brain structure in schizophrenia and bipolar disorder. Transl Psychiatry. 2019;9:12.

3. Clementz BA, Sweeney JA, Hamm JP, Ivleva El, Ethridge LE, Pearlson GD, et al. Identification of distinct psychosis biotypes using brain-based biomarkers. Am J Psychiatry. 2016;173:373-84.

4. Ivleva El, Bidesi AS, Keshavan MS, Pearlson GD, Meda SA, Dodig D, et al. Gray matter volume as an intermediate phenotype for psychosis: BipolarSchizophrenia Network on Intermediate Phenotypes (B-SNIP). Am J Psychiatry. 2013;170:1285-96.

5. Arnold SJ, Ivleva El, Gopal TA, Reddy AP, Jeon-Slaughter $H$, Sacco $C B$, et al. Hippocampal volume is reduced in schizophrenia and schizoaffective disorder but not in psychotic bipolar I disorder demonstrated by both manual tracing and automated parcellation (FreeSurfer). Schizophr Bull. 2015;41:233-49.

6. Meda SA, Wang Z, Ivleva El, Poudyal G, Keshavan MS, Tamminga CA, et al. Frequency-specific neural signatures of spontaneous low-frequency resting state fluctuations in psychosis: evidence from Bipolar-Schizophrenia Network on Intermediate Phenotypes (B-SNIP) Consortium. Schizophr Bull. 2015; 41:1336-48

7. Lui S, Yao L, Xiao Y, Keedy SK, Reilly JL, Keefe RS, et al. Resting-state brain function in schizophrenia and psychotic bipolar probands and their first-degree relatives. Psychol Med. 2015;45:97-108.

8. Meda SA, Ruano G, Windemuth A, O'Neil K, Berwise C, Dunn SM, et al. Multivariate analysis reveals genetic associations of the resting default mode network in psychotic bipolar disorder and schizophrenia. Proc Natl Acad Sci USA. 2014;111: E2066-75.

9. Friston KJ. The disconnection hypothesis. Schizophr Res. 1998;30:115-25.

10. Fornito A, Zalesky A, Breakspear M. The connectomics of brain disorders. Nat Rev Neurosci. 2015;16:159-72.

11. Coyle JT, Balu DT, Puhl MD, Konopaske GT. History of the concept of disconnectivity in schizophrenia. Harv Rev Psychiatry. 2016;24:80-6.

12. Fornito A, Zalesky A, Pantelis C, Bullmore ET. Schizophrenia, neuroimaging and connectomics. Neuroimage. 2012;62:2296-314.

13. Farahani FV, Karwowski W, Lighthall NR. Application of graph theory for identifying connectivity patterns in human brain networks: a systematic review. Front Neurosci. 2019;13:585

14. Bullmore E, Sporns O. Complex brain networks: graph theoretical analysis of structural and functional systems. Nat Rev Neurosci. 2009;10:186-98.

15. Schmidt A, Diwadkar VA, Smieskova R, Harrisberger F, Lang UE, McGuire $P$, et al. Approaching a network connectivity-driven classification of the psychosis continuum: a selective review and suggestions for future research. Front Hum Neurosci. 2014;8:1047

16. Sheffield JM, Kandala S, Tamminga CA, Pearlson GD, Keshavan MS, Sweeney JA, et al. Transdiagnostic associations between functional brain network integrity and cognition. JAMA Psychiatry. 2017;74:605-13.

17. Rubinov M, Bullmore E. Schizophrenia and abnormal brain network hubs. Dialogues Clin Neurosci. 2013;15:339-49.

18. Yan H, Tian L, Wang Q, Zhao Q, Yue W, Yan J, et al. Compromised small-world efficiency of structural brain networks in schizophrenic patients and their unaffected parents. Neurosci Bull. 2015;31:275-87.

19. Schmidt A, Crossley NA, Harrisberger F, Smieskova R, Lenz C, Riecher-Rossler A, et al. Structural network disorganization in subjects at clinical high risk for psychosis. Schizophr Bull. 2017;43:583-91.

20. Gong Q, Lui S, Sweeney JA. A selective review of cerebral abnormalities in patients with first-episode schizophrenia before and after treatment. Am J Psychiatry. 2016;173:232-43.

21. Abi-Dargham A, Horga $G$. The search for imaging biomarkers in psychiatric disorders. Nat Med. 2016;22:1248-55.

22. Zalesky A, Fornito A, Seal ML, Cocchi L, Westin CF, Bullmore ET, et al. Disrupted axonal fiber connectivity in schizophrenia. Biol Psychiatry. 2011;69:80-9.

23. Morgan SE, Seidlitz J, Whitaker KJ, Romero-Garcia R, Clifton NE, Scarpazza C, et al. Cortical patterning of abnormal morphometric similarity in psychosis is associated with brain expression of schizophrenia-related genes. Proc Natl Acad Sci USA. 2019;116:9604-09.

24. Alexander-Bloch A, Giedd JN, Bullmore E. Imaging structural co-variance between human brain regions. Nat Rev Neurosci. 2013;14:322-36.

25. Seidlitz J, Vasa F, Shinn M, Romero-Garcia R, Whitaker KJ, Vertes PE, et al. Morphometric similarity networks detect microscale cortical organization and predict inter-individual cognitive variation. Neuron. 2018;97:231-47.e7.

26. He Y, Chen ZJ, Evans AC. Small-world anatomical networks in the human brain revealed by cortical thickness from MRI. Cereb Cortex. 2007:17:2407-19.
27. Bassett DS, Bullmore E, Verchinski BA, Mattay VS, Weinberger DR, MeyerLindenberg A. Hierarchical organization of human cortical networks in health and schizophrenia. J Neurosci. 2008;28:9239-48.

28. Zhang $Y$, Lin L, Lin $C P$, Zhou $Y$, Chou KH, Lo CY, et al. Abnormal topological organization of structural brain networks in schizophrenia. Schizophr Res. 2012;141:109-18.

29. Bhojraj TS, Prasad KM, Eack SM, Francis AN, Montrose DM, Keshavan MS. Do interregional gray-matter volumetric correlations reflect altered functional connectivity in high-risk offspring of schizophrenia patients? Schizophr Res. 2010;118:62-8.

30. Shi F, Yap PT, Gao W, Lin W, Gilmore JH, Shen D. Altered structural connectivity in neonates at genetic risk for schizophrenia: a combined study using morphological and white matter networks. Neuroimage. 2012;62:1622-33.

31. Hill SK, Reilly JL, Keefe RS, Gold JM, Bishop JR, Gershon ES, et al. Neuropsychological impairments in schizophrenia and psychotic bipolar disorder: findings from the Bipolar-Schizophrenia Network on Intermediate Phenotypes (B-SNIP) study. Am J Psychiatry. 2013;170:1275-84.

32. Reininghaus U, Bohnke JR, Chavez-Baldini U, Gibbons R, Ivleva E, Clementz BA et al. Transdiagnostic dimensions of psychosis in the Bipolar-Schizophrenia Network on Intermediate Phenotypes (B-SNIP). World Psychiatry. 2019;18:67-76.

33. Ivleva El, Clementz BA, Dutcher AM, Arnold SJM, Jeon-Slaughter H, Aslan S, et al. Brain structure biomarkers in the psychosis biotypes: findings from the bipolarschizophrenia network for intermediate phenotypes. Biol Psychiatry. 2017;82:26-39.

34. Doherty JL, Owen MJ. Genomic insights into the overlap between psychiatric disorders: implications for research and clinical practice. Genome Med. 2014;6:29.

35. Tamminga CA, Ivleva El, Keshavan MS, Pearlson GD, Clementz BA, Witte B, et al Clinical phenotypes of psychosis in the bipolar-schizophrenia network on intermediate phenotypes (B-SNIP). Am J Psychiatry. 2013;170:1263-74.

36. Tijms BM, Series $P$, Willshaw DJ, Lawrie SM. Similarity-based extraction of individual networks from gray matter MRI scans. Cereb Cortex. 2012;22:1530-41.

37. Evans AC. Networks of anatomical covariance. Neuroimage. 2013;80:489-504.

38. Weese J, Rosch P, Netsch T, Blaffert T, Quist M. Gray-value based registration of CT and MR images by maximization of local correlation. In: Taylor C, Colchester A, editors. Medical Image Computing and Computer-Assisted Intervention, Miccai'99, Proceedings. Berlin, Heidelberg: Springer; 1999. p. 656-63.

39. van Wijk BCM, Stam CJ, Daffertshofer A. Comparing brain networks of different size and connectivity density using graph theory. PLoS ONE. 2010;5:e13701.

40. Batalle D, Munoz-Moreno E, Figueras F, Bargallo N, Eixarch E, Gratacos E. Normalization of similarity-based individual brain networks from gray matter MR and its association with neurodevelopment in infants with intrauterine growth restriction. Neuroimage. 2013;83:901-11.

41. Tzourio-Mazoyer N, Landeau B, Papathanassiou D, Crivello F, Etard O, Delcroix N, et al. Automated anatomical labeling of activations in SPM using a macroscopic anatomical parcellation of the MNI MRI single-subject brain. Neuroimage. 2002;15:273-89.

42. Watts DJ, Strogatz SH. Collective dynamics of 'small-world' networks. Nature. 1998;393:440-2.

43. Zhang J, Wang J, Wu Q, Kuang W, Huang X, He Y, et al. Disrupted brain connectivity networks in drug-naive, first-episode major depressive disorder. Biol Psychiatry. 2011;70:334-42.

44. Achard S, Bullmore E. Efficiency and cost of economical brain functional net works. PLoS Comput Biol. 2007;3:e17.

45. Latora V, Marchiori M. Efficient behavior of small-world networks. Phys Rev Lett. 2001;87:17.

46. Menon V. Large-scale brain networks and psychopathology: a unifying triple network model. Trends Cogn Sci. 2011;15:483-506.

47. Patel R, Spreng RN, Shin LM, Girard TA. Neurocircuitry models of posttraumatic stress disorder and beyond: a meta-analysis of functional neuroimaging studies. Neurosci Biobehav Rev. 2012;36:2130-42.

48. Buckner RL, Andrews-Hanna JR, Schacter DL. The brain's default network: anatomy, function, and relevance to disease. Ann N. Y Acad Sci. 2008;1124:1-38.

49. Gusnard DA, Raichle ME, Raichle ME. Searching for a baseline: functional imaging and the resting human brain. Nat Rev Neurosci. 2001;2:685-94.

50. Zalesky A, Fornito A, Bullmore ET. Network-based statistic: identifying differences in brain networks. Neuroimage. 2010;53:1197-207.

51. Kay SR, Fiszbein A, Opler LA. The positive and negative syndrome scale (PANSS) for schizophrenia. Schizophr Bull. 1987;13:261-76.

52. Young RC, Biggs JT, Ziegler VE, Meyer DA. A rating scale for mania: reliability, validity and sensitivity. Br J Psychiatry. 1978;133:429-35.

53. Montgomery SA, Asberg M. A new depression scale designed to be sensitive to change. Br J Psychiatry. 1979;134:382-9.

54. Martin A, Chao LL. Semantic memory and the brain: structure and processes. Curr Opin Neurobiol. 2001;11:194-201. 
55. Kiehl KA, Smith AM, Mendrek A, Forster BB, Hare RD, Liddle PF. Temporal lobe abnormalities in semantic processing by criminal psychopaths as revealed by functional magnetic resonance imaging. Psychiatry Res. 2004;130:297-312.

56. Liu $Y$, Liang $M$, Zhou $Y$, He $Y$, Hao $Y$, Song $M$, et al. Disrupted small-world networks in schizophrenia. Brain. 2008;131:945-61.

57. Alexander-Bloch AF, Gogtay N, Meunier D, Birn R, Clasen L, Lalonde F, et al. Disrupted modularity and local connectivity of brain functional networks in childhood-onset schizophrenia. Front Syst Neurosci. 2010;4:147.

58. Honea R, Crow TJ, Passingham D, Mackay CE. Regional deficits in brain volume in schizophrenia: a meta-analysis of voxel-based morphometry studies. Am J Psychiatry. 2005; 162:2233-45.

59. Walton E, Hibar DP, van Erp TG, Potkin SG, Roiz-Santianez R, Crespo-Facorro B, et al. Positive symptoms associate with cortical thinning in the superior temporal gyrus via the ENIGMA Schizophrenia consortium. Acta Psychiatr Scand. 2017;135:439-47.

60. Zhang W, Li S, Wang X, Gong Y, Yao L, Xiao Y, et al. Abnormal dynamic functional connectivity between speech and auditory areas in schizophrenia patients with auditory hallucinations. Neuroimage Clin. 2018;19:918-24.

61. Allen P, Modinos G, Hubl D, Shields G, Cachia A, Jardri R, et al. Neuroimaging auditory hallucinations in schizophrenia: from neuroanatomy to neurochemistry and beyond. Schizophr Bull. 2012;38:695-703.

62. Ellison-Wright I, Bullmore E. Anatomy of bipolar disorder and schizophrenia: a meta-analysis. Schizophr Res. 2010;117:1-12.

63. Crossley NA, Mechelli A, Fusar-Poli P, Broome MR, Matthiasson P, Johns LC, et al. Superior temporal lobe dysfunction and frontotemporal dysconnectivity in subjects at risk of psychosis and in first-episode psychosis. Hum Brain Mapp. 2009;30:4129-37.

64. Mechelli A, Riecher-Rossler A, Meisenzahl EM, Tognin S, Wood SJ, Borgwardt SJ, et al. Neuroanatomical abnormalities that predate the onset of psychosis: a multicenter study. Arch Gen Psychiatry. 2011;68:489-95.

65. Allen $P$, Chaddock CA, Howes OD, Egerton A, Seal ML, Fusar-Poli $P$, et al. Abnormal relationship between medial temporal lobe and subcortical dopamine function in people with an ultra high risk for psychosis. Schizophr Bull. 2012;38:1040-9.

66. Tijms BM, Sprooten E, Job D, Johnstone EC, Owens DG, Willshaw D, et al. Grey matter networks in people at increased familial risk for schizophrenia. Schizophr Res. 2015;168:1-8.

67. Fusar-Poli P, Borgwardt S, Crescini A, Deste G, Kempton MJ, Lawrie S, et al. Neuroanatomy of vulnerability to psychosis: a voxel-based meta-analysis. Neurosci Biobehav Rev. 2011;35:1175-85.

68. Brent BK, Thermenos HW, Keshavan MS, Seidman LJ. Gray matter alterations in schizophrenia high-risk youth and early-onset schizophrenia: a review of structural MRI findings. Child Adolesc Psychiatr Clin N. Am. 2013;22:689-714.

69. Allen P, Moore H, Corcoran CM, Gilleen J, Kozhuharova P, Reichenberg A, et al. Emerging temporal lobe dysfunction in people at clinical high risk for psychosis. Front Psychiatry. 2019;10:298.

70. Benetti S, Mechelli A, Picchioni M, Broome M, Williams S, McGuire P. Functional integration between the posterior hippocampus and prefrontal cortex is impaired in both first episode schizophrenia and the at risk mental state. Brain. 2009:132:2426-36.

71. Lo CY, Su TW, Huang CC, Hung CC, Chen WL, Lan TH, et al. Randomization and resilience of brain functional networks as systems-level endophenotypes of schizophrenia. Proc Natl Acad Sci USA. 2015;112:9123-8.

72. Goldman AL, Pezawas L, Mattay VS, Fischl B, Verchinski BA, Chen Q, et al. Widespread reductions of cortical thickness in schizophrenia and spectrum disorders and evidence of heritability. Arch Gen Psychiatry. 2009;66:467-77.

73. McDonald C, Marshall N, Sham PC, Bullmore ET, Schulze K, Chapple B, et al. Regional brain morphometry in patients with schizophrenia or bipolar disorder and their unaffected relatives. Am J Psychiatry. 2006;163:478-87.

74. Lui S, Li T, Deng W, Jiang L, Wu Q, Tang H, et al. Short-term effects of antipsychotic treatment on cerebral function in drug-naive first-episode schizophrenia revealed by "resting state" functional magnetic resonance imaging. Arch Gen Psychiatry. 2010;67:783-92.

75. Keedy SK, Reilly JL, Bishop JR, Weiden PJ, Sweeney JA. Impact of antipsychotic treatment on attention and motor learning systems in first-episode schizophrenia. Schizophr Bull. 2015;41:355-65.

76. Meda SA, Clementz BA, Sweeney JA, Keshavan MS, Tamminga CA, Ivleva El, et al. Examining functional resting-state connectivity in psychosis and its subgroups in the Bipolar-Schizophrenia Network on Intermediate Phenotypes Cohort. Biol Psychiatry Cogn Neurosci Neuroimaging. 2016;1:488-97.

77. Cardno AG, Rijsdijk FV, Sham PC, Murray RM, McGuffin P. A twin study of genetic relationships between psychotic symptoms. Am J Psychiatry. 2002;159:539-45.

78. Mancuso SG, Morgan VA, Mitchell PB, Berk M, Young A, Castle DJ. A comparison of schizophrenia, schizoaffective disorder, and bipolar disorder: results from the Second Australian national psychosis survey. J Affect Disord. 2015;172:30-7.

79. Tijms BM, Kate MT, Wink AM, Visser PJ, Ecay M, Clerigue $M$, et al. Gray matter network disruptions and amyloid beta in cognitively normal adults. Neurobiol Aging. 2016;37:154-60.

80. Tijms BM, Moller C, Vrenken H, Wink AM, de Haan W, van der Flier WM, et al. Single-subject grey matter graphs in Alzheimer's disease. PLoS ONE. 2013;8: e58921.

Open Access This article is licensed under a Creative Commons Attribution 4.0 International License, which permits use, sharing, adaptation, distribution and reproduction in any medium or format, as long as you give appropriate credit to the original author(s) and the source, provide a link to the Creative Commons license, and indicate if changes were made. The images or other third party material in this article are included in the article's Creative Commons license, unless indicated otherwise in a credit line to the material. If material is not included in the article's Creative Commons license and your intended use is not permitted by statutory regulation or exceeds the permitted use, you will need to obtain permission directly from the copyright holder. To view a copy of this license, visit http://creativecommons. org/licenses/by/4.0/.

(c) The Author(s) 2019 